\title{
The E-cigarette - a New Tool to Aid Smoking Cessation
}

\author{
Pierre Bartsch \\ Department of Chest Diseases, University of Liège, Belgium
}

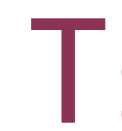

his editorial shows that, despite controversies in the public health community and among professionals regarding the role of e-cigarettes as an aid to stopping smoking, and although the potential health risks of long-term use of nicotine are unknown, modern devices should be regarded more as a solution than a problem. Clinicians, owing to the high morbidity and mortality of smoking should include e-cigarettes as efficient tools to help smokers to quit smoking or at least as a substitute for cigarettes. Effort is needed to improve knowledge about this tool.

DOI: https://doi.org/10.17925/ERPD.2017.03.01.19

\section{Keywords}

Smoking cessation, e-cigarettes, tobacco harm reduction

Disclosure: Pierre Bartsch has no conflicts of interest to declare in relation to this article. No funding was received for the publication of this article.

Authorship: All named authors meet the International Committee of Medical Journal Editors (ICMJE) criteria for authorship of this manuscript, take responsibility for the integrity of the work as a whole, and have given final approval for the version to be published.

open Access: This article is published under the Creative Commons Attribution Noncommercial License, which permits any non-commercial use, distribution, adaptation and reproduction provided the original author(s) and source are given appropriate credit.

Received: 31 January 2017

Published Online: 14 April 2017

Citation: EU Respiratory \& Pulmonary Diseases, 2017;3(1):19-20

Corresponding Author: Pierre Bartsch, Domaine Universitaire du Sart, Tilman B35 4000, Liège, Belgium. E: pierre.bartsch@ulg.ac.be
A new social phenomenon has occurred since the end of the first decade of the 21st century, namely, the rise of the electronic cigarette (e-cig) also called the electronic nicotine delivery system (ENDS). The two names are not strictly equivalent, as the latter implies the presence of nicotine.

As a lung physician confronted daily with the difficult challenge of smoking cessation, in this article, I will consider only the nicotine-containing devices. This is a social issue as neither pharmaceutical industry, nor the tobacco industry predicted that e-cigs would become so popular in the absence of big advertising campaigns. The first models looked like cigarettes (cigalikes) and appeared more as gadgets than as a possible tool for smokers wanting to quit smoking. The inventor, a chinese person named Hon Lik was nevertheless pushed to design a product delivering nicotine without dangerous components, after witnessing his father dying from a lung cancer. We do not know if this pharmacist and engineer was aware of Russel's claim that 'people smoke for nicotine but they die from tar'.1

Health professionals were not the initiators of this way of stopping or reducing smoking, the initiative came from the population of smokers itself. The Public Health community, all over the world, was, and still is, very reluctant to endorse the practice. The ideological reason is an opposition to the concept of harm reduction itself. Tobacco harm reduction (THR) is considered by opponents as compromising smoking cessation constituting a gateway to smoking among nonsmoking adolescents. The real-world situation is the $80 \%$ failure of abstinence with the current approved treatments of nicotine dependence in the general population. So if electronic cigarettes are at least as efficient as nicotine patches for smoking cessation, we should utilise them for this indication, if they are proven to be not at all, or much less dangerous for health, which seems to be the case.?

Many surveys show that a number of users who quit will continue to 'vape', which is considered a safer way, not to smoke, but to vaporise nicotine. For those people, we are in the THR situation A more scientific reason to contest the place of e-cigs in smoking cessation is still the absence of large randomised controlled trials. The Cochrane Review on the subject ${ }^{3}$ states that electronic cigarettes may have the same efficacy as the nicotine patch, and with a short time use of up to two years, not seeming to be deleterious for human health.

Today, if cigalikes (first generation e-cigarettes) are no longer regarded as nicotine substitutes, this is not the case for the second and, mostly, third generation e-cigs, which deliver nicotine to the brain almost as fast as a classical cigarette. ${ }^{4}$ A population study of smokers, not using smoking cessation help from health professionals (HP), showed a much better success with e-cigs, than with nicotine replacement therapy (NRT) such as patches bought over the counter.

Therefore, when a HP is confronted to a smoker unable or unwilling to quit after using licensed medicines, it is mandatory to encourage the use of last-generation electronic cigarettes. This is a challenge not only for lung physicians, cardiologists, oncologists and other specialists, 
but also for general practitioners, who have a duty to improve their knowledge about the e-cigs and e-liquids filling the numerous devices of unequal quality on the market.

In Belgium, during the formation delivered to HP specialising in smoking cessation, "tobaccologists", we include lectures and workshops about nicotine containing e-cigarettes, now legal, as a tool for the smokers who have failed with licensed products for stopping smoking. ${ }^{6}$ The policy of including electronic cigarettes as a tool in the smoking cessation process is a cornerstone of Public Health England, ${ }^{7}$ which has also published guidelines on practical issues regarding e-cigarette use. ${ }^{8}$

This document concludes that 'use of e-cigarettes by never smokers remains negligible and evidence does not support the view that e-cigarettes are acting as a gateway into smoking among young people'. ${ }^{8}$ To leave the discussion open on this controversial issue, a report about the 2014 National Youth Tobacco Survey (NYTS) in the US, stresses the complexity of defining patterns of frequency, temporality and poly-use in youth (cigarette, cigar, smokeless tobacco, e-cigarette). They ask for several waves of longitudinal surveys before closing the question. ${ }^{9}$

Regarding smoking cessation, even if we still do not have robust evidence-based proof of efficacy, there is a huge amount of positive observation from millions of ex-smokers, driving our decision to recommend e-cigarettes to smokers unable to sustain abstinence with available medications and strategies. A return to breathing only air in the vast population of satisfied vapers will be a challenge for the future, as vaping is not the ultimate goal for optimal health. $\square$
1. Russell MA, Low-tar medium-nicotine cigarettes: a new approach to safer smoking, Br Med J, 1976;1:1430-3.
Nutt DJ, Phillips LD, Balfour D, et al., Estimating the harms of Nutt DJ, Phillips $L D$, Balfour D, et al., Estimating the harms
nicotine-containing products using the MCDA approach, Eur Addict Res, 2014:20:218-25.

3. Hartmann-Boyce J, McRobbie H, Bullen C, et al., Electronic Hartmante-Boyce J, McRobbie $\mathrm{H}$, Bullen C, et al., Electronic
cigarettes for smoking cessation, Cochrane Database Syst Rev 2016;9:CD010216.

4. Wagener TL, Floyd EL, Stepanov I, et al., How combustible cigarettes met their match? The nicotine delivery profiles cigarettes met their match? The nicotine delivery profiles and third-generation electronic cigarette users, Tob Control $2017 \cdot 26 \cdot 023-8$

5. Brown J, Beard E, Kotz D, et al., Real-world effectiveness of e-cigarettes when used to aid smoking cessation: a . 6. Bartsch $P$, Delvaux $M$, Englebert $\mathrm{E}$, et al., cigarettes électroniques et arrêt du tabac: la situation en Belgique, 2017:72:114-19.

7. Public Health England. E-cigarettes: an evidence update (Public Health 2015). Available at: umw gov uk/government/uploads/ Health 2015). Available at: www.gov.uk/government/uploads/
system/uploads/attachment_data/ile/457102/Ecigarettes_an
evidence_update_A_report_commissioned_by Public_Health England_FINAL.pdf (accessed 7 February 2017).

8. McEwen A, McRobbie H, Electronic cigarettes: a briefing for stop smoking services, National Centre for Smoking Cessatio and Training, 2016. Available at. Www. ncsct.co.uk/ust/pub/ Electronic_cigarettes._A_briefing for pdf (accessed 7 February 2017).

Villanti AC, Pearson JL, Glasser AM, et al., Frequency of youth e-cigarettes and tobacco use patterns in the US: measureme 2016: pii:ntw388, doi: 10.1093/ntr/ntw388 